\title{
Weibull Inverse Lomax Distribution
}

Amal Soliman Hassan

Mathematical Statistics, Cairo University, Faculty of Graduate Studies for Statistical Research, Giza, Egypt

dr.amalelmoslamy@gmail.com

Rokaya Elmorsy Mohamed

Mathematical Statistics, Cairo University, Faculty of Graduate Studies for Statistical Research, Giza, Egypt

rokayaelmorsy@gmail.com

\begin{abstract}
A four-parameter lifetime model, named as Weibull inverse Lomax (WIL) is presented and studied. Some structural properties are derived. The estimation of the model parameters is performed based on Type II censored samples. Maximum likelihood estimators along with asymptotic confidence intervals of population parameters and reliability function are constructed. The property of consistency of maximum likelihood estimators is verified on the basis of simulated samples. Further, the results are applied on two real data.
\end{abstract}

Keywords: Inverse Lomax distribution, Weibull-G family, Maximum likelihood estimation, Type II censored sample.

\section{Introduction}

In the past, a number of probability distributions has been found to be useful in the fields of insurance, engineering, medicine, economics and finance (among others). However, generalizing these probability distributions provided several new models that are more flexible compared to the baseline distributions. Several attempts have been made to extend new families of distributions by utilizing a number of techniques. One may refer to Eugene et al.( 2002), Jones (2004), Alzaatreh et al. (2013) and Bourguignon et al. (2014), for some more interesting results on this topic. Out of the several families of distributions, of interest to us in this study is the Weibull- generated (W-G) family of distributions presented by Bourguignon et al. (2014). The cumulative distribution function (cdf) of the $\mathrm{W}-\mathrm{G}$ is defined as follows,

$$
F(x ; a, b, \zeta)=\int_{0}^{\overline{G(x ; \zeta)}} a b t^{b-1} e^{-a t^{b}} d t=1-e^{-a\left[\frac{G(x ; \zeta)}{\bar{G}(x ; \zeta)}\right]^{b}},
$$

where, $G(x ; \zeta)$ is the baseline cdf with parameter vector $\zeta$ and $\bar{G}(x ; \zeta)=1-G(x ; \zeta)$. Based on W-G family, some particular distributions have been studied by several authors; see for example; Tahir et al. (2015), Merovci and Elbatal (2015), Hassen et al. (2016), Abouelmagd et al. (2017) and Ibrahim et al. (2017).

The inverse Lomax (IL) is belonging to the family of generalized beta distribution. The IL distribution is the inverse of Lomax distribution. Kleiber and Kotz (2003) showed that the IL distribution can be used in economics and actuarial sciences. The IL distribution has a lot of applications in stochastic modeling of decreasing failure rate life components 
and life testing. Kleiber (2004) used the IL distribution to get Lorenz ordering relationship among ordered statistics. According to McKenzie et al. (2011), the IL distribution has been applied on geophysical databases. Rahman et al. (2013) discussed the estimated and predicted values using Bayesian approach under various loss functions. Rahman and Aslam (2014) used two-component mixture IL model for the prediction of future ordered observations in Bayesian framework using predictive model. Yadav et al. (2016) discussed the parameter estimation of the IL distribution based on hybrid censored samples. Rahman and Aslam (2017) introduced an estimation of two-component mixture IL model via Bayesian approach. Singh et al. (2016) calculated the reliability estimators of IL distribution under Type II censoring. Bayesian estimation of two-component mixture of IL distribution based on Type-I censoring scheme was discussed by Reyad and Othman (2018).

The probability density function (pdf) of two-parameter IL distribution is specified by

$$
g(x ; \lambda, \beta)=\frac{\lambda}{\beta^{\lambda}} x^{\lambda-1}\left(1+\frac{x}{\beta}\right)^{-(\lambda+1)}, \quad \quad \beta, \lambda, x>0,
$$

where, $\beta$ and $\lambda$ are the scale and shape parameters respectively. The cdf corresponding to $(2)$ is

$$
G(x ; \lambda, \beta)=\left(1+\frac{\beta}{x}\right)^{-\lambda}, \quad \quad \beta, \lambda, x>0 .
$$

It is common practice in a life testing experiment to terminate the experiment before all the units have failed as there is limited availability of test time. The observations obtained in such situation are called censored samples. Censoring is done to save time and cost associated with testing. Type-I and Type-II censoring (TIIC) schemes are the two most popular censoring schemes used in the reliability and life testing experiments. In TIIC scheme, the experiment of $n$ items is placed on test and the number of uncensored data $r$ is predetermined. Instead of continuing experiments until all $n$ items have failed, the experiment is terminated when the $r^{\text {th }}$ item fails. The remaining $n-r$ items are regarded as censored data.

The main aim here is to introduce a four-parameter Weibull inverse Lomax (WIL) distribution as well as study the estimate of its population parameters depending on TIIC samples. The rest of the paper contains the following sections. In Section 2, the pdf, cdf, reliability function, hazard rate function and reversed hazard rate function of the WIL distribution are defined. Statistical properties of the WIL distribution include; moments, Rényi entropy, quantile function and distribution of order statistics are derived in Section 3. The maximum likelihood (ML) estimators of the model parameters are derived in Section 4. Simulation study is carried out in Section 5. Analysis to real data sets is presented in Section 6. The article ends with concluding remarks.

\section{Weibull Inverse Lomax Distribution}

In this section, the WIL distribution is defined depending on the W-G family. The expansion for the WIL density function is provided. The reliability characterizations of WIL distribution are provided.

Taking the base-line distribution in (1) to be the cdf of IL defined in (3), hence the cdf of WIL distribution is specified by 


$$
F(x ; \psi)=1-e^{-a\left[\left(1+\frac{\beta}{x}\right)^{\lambda}-1\right]^{-b}}, a, b, \lambda, \beta, x>0,
$$

where, $\beta, a$ are the scale parameters, $b, \lambda$ are the shape parameters and $\psi=(\lambda, a, b, \beta)$ is set of parameters. The pdf of the WIL distribution corresponding to (4) is specified by $f(x ; \psi)=a b \lambda \beta^{-\lambda b} x^{\lambda b-1}\left(1+\frac{x}{\beta}\right)^{-(\lambda b+1)}\left(1-\left(1+\frac{\beta}{x}\right)^{-\lambda}\right)^{-(b+1)} e^{-a\left[\left(1+\frac{\beta}{x}\right)^{\lambda}-1\right]^{-b}} a, b, \lambda, \beta, x>0$.

For $b=2$, the pdf (5) reduces to Rayleigh inverse Lomax distribution as a new model. For $b=1$, the pdf (5) reduces to exponential inverse Lomax distribution as a new model. A random variable $X$ with density function (5) will be denoted by $X \sim \mathrm{WIL}(x ; \psi)$.

\subsection{Useful Expansion}

Here, an explicit expression for the WIL pdf is provided. By using the exponential function in (5), we obtain

$$
f(x ; \psi)=b \lambda \beta^{-\lambda b} \sum_{i=0}^{\infty} \frac{(-1)^{i} a^{i+1}}{i !} x^{\lambda b-1}\left(1+\frac{x}{\beta}\right)^{-(\lambda b+1)}\left(1+\frac{\beta}{x}\right)^{-\lambda b i}\left(1-\left(1+\frac{\beta}{x}\right)^{-\lambda}\right)^{-(b+b i+1)} .
$$

Since, the binomial expansion, for real non-integer value of $k$, is given

$$
(1-z)^{-k}=\sum_{j=0}^{\infty} \frac{\Gamma(k+j) z^{j}}{\Gamma(k) j !},|z|<1, k>0 \text {. }
$$

Then, by applying the binomial expansion (7) in (6), then we have

$f(x ; \psi)=b \lambda \sum_{i, j=0}^{\infty} \frac{(-1)^{i} a^{i+1} \Gamma(b+b i+1+j) \beta^{-\lambda(b+b i+j)}}{\Gamma(b+b i+1) i ! j !} x^{\lambda(b+b i+j)-1}\left(1+\frac{x}{\beta}\right)^{-(\lambda(b+b i+j)+1)}$.

Or, it can be written as follows

$$
\begin{gathered}
f(x ; \psi)=\sum_{i, j=0}^{\infty} w_{i, j} g_{\lambda(b+b i+j), \beta}(x), \\
w_{i, j}=\frac{(-1)^{i} a^{i+1} b \Gamma(b+b i+j)}{\Gamma(b+b i+1) i ! j !}
\end{gathered}
$$

where $g_{\lambda(b+b i+j), \beta}(x)$ denotes the pdf of IL distribution with parameters $\lambda(b+b i+j)$ and $\beta$.

\subsection{Reliability Characterization}

The reliability function and hazard rate function (hrf) of $X$ are given, respectively, as follows:

$$
R(x ; \psi)=\exp -a\left[\left(1+\frac{\beta}{x}\right)^{\lambda}-1\right]^{-b}
$$


and

$h(x ; \psi)=a b \lambda \beta^{-\lambda b} x^{\lambda b-1}\left(1+\frac{x}{\beta}\right)^{-(\lambda b+1)}\left(1-\left(1+\frac{\beta}{x}\right)^{-\lambda}\right)^{-(b+1)}$.

Further, the reversed hrf $(r(x ; \psi))$ and cumulative hrf $(H(x ; \psi))$ of $X$ are obtained as follows

$r(x ; \psi)=a b \lambda \beta^{-\lambda b} x^{\lambda b-1}\left(1+\frac{x}{\beta}\right)^{-(\lambda b+1)}\left(1-\left(1+\frac{\beta}{x}\right)^{-\lambda}\right)^{-(b+1)} e^{-a\left[\left(1+\frac{\beta}{x}\right)^{\lambda}-1\right]^{-b}}\left(1-e^{-a\left[\left(1+\frac{\beta}{x}\right)^{\lambda}-1\right]^{-b}}\right)^{-1}$,

and,

$H(x ; \psi)=-\ln (R(x ; \psi))=a\left[\left(1+\frac{\beta}{x}\right)^{\lambda}-1\right]^{-b}$.

Plots of the pdf and hrf of the WIL distribution are displayed in Figures 1 and 2, respectively, for different values of parameters. As seen the shapes of pdf take different forms as symmetric, right skewed, and unimodel. Also, it is clear from Figure 2 that the shapes of the hrf are J-shaped, reversed $\mathrm{J}$ - shaped, decreasing and increasing at some selected values of parameters.

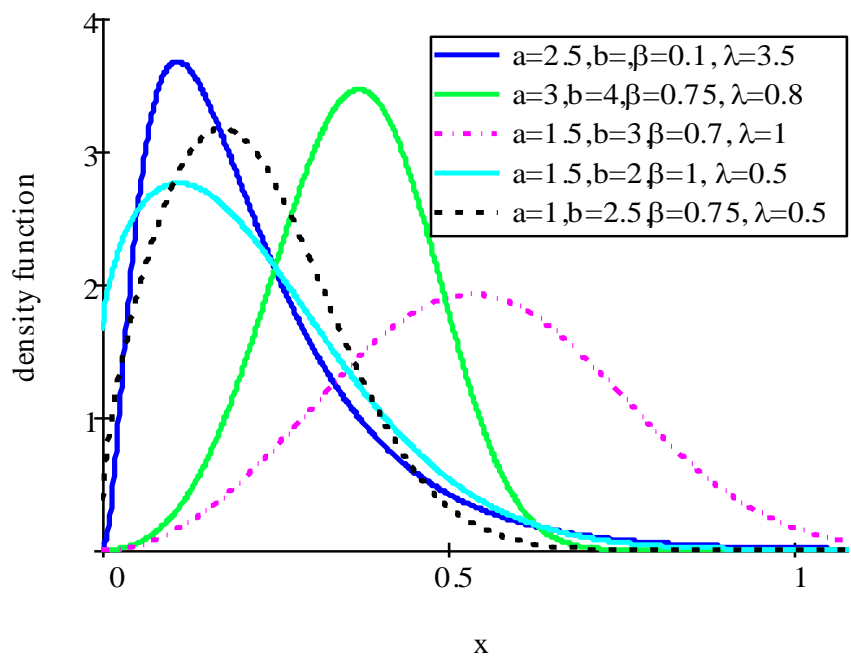

Figure 1: The pdf of the WIL distribution for some parameter values

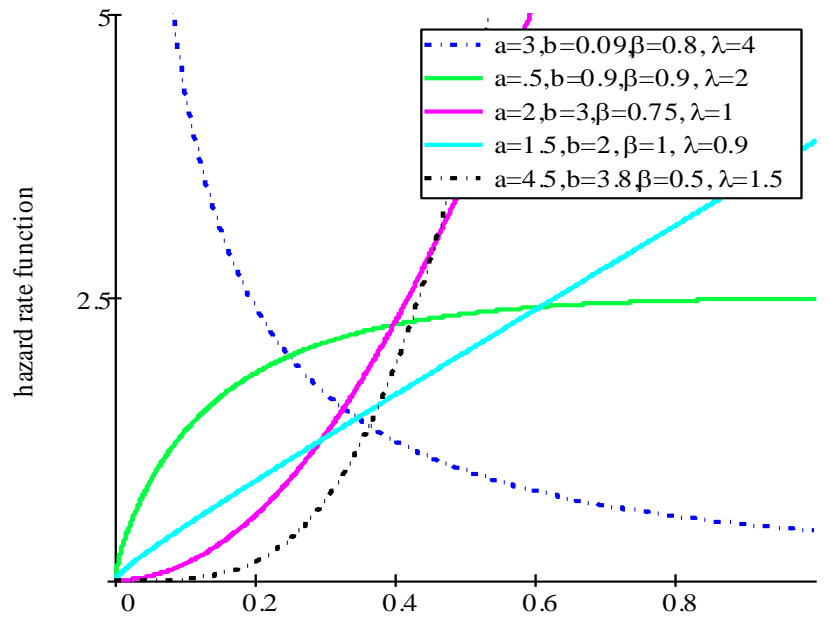

$\mathrm{X}$

Figure 2: The hrf of the WIL distribution for some parameter values

\section{Statistical Properties}

\subsection{Moments and Inverse Moments}

The $s^{\text {th }}$ moment of the WIL distribution about zero is derived by using pdf (9) as follows $\mu_{s}^{\prime}=\int_{0}^{\infty} x^{s} \sum_{i, j=0}^{\infty} w_{i, j} g_{\lambda(b+b i+j), \beta}(x) d x$. 
Therefore, the $s^{\text {th }}$ moment of the WIL distribution can be obtained as follows

$\mu_{s}^{\prime}=\sum_{i, j=0}^{\infty} w_{i, j} \beta^{s} \frac{\Gamma(1-s) \Gamma(s+\lambda(b+b i+j))}{\Gamma(\lambda(b+b i+j))}, \quad s=1,2, \ldots$,

where, $\Gamma(0)=-\gamma, \Gamma(-s)=\frac{(-1)^{s}}{s !} \varphi(s)-\frac{(-1)^{s}}{s !} \gamma$ for $s=1,2, \ldots, \gamma$ denotes Euler's constant and $\varphi(s)=\sum_{i=1}^{s} \frac{1}{i}$ ( see Fisher and Kilicman, 2012). The mean of $X$ follows by setting $s=1$ in $(10)$ as

$$
\mu_{1}^{\prime}=\sum_{i, j=0}^{\infty} \frac{(-a)^{i+1} b \Gamma(b+b i+j+1) \beta \gamma}{\Gamma(b+b i+1) i ! j !},
$$

where, $\gamma$ is Euler's constant. The $s^{\text {th }}$ central moment $\left(\mu_{s}\right)$ of $X$ is given by

$$
\mu_{s}=E\left(X-\mu_{1}^{\prime}\right)^{s}=\sum_{i=0}^{s}(-1)^{i}\left(\begin{array}{l}
s \\
i
\end{array}\right)\left(\mu_{1}^{\prime}\right)^{i} \mu_{s-i}^{\prime} \text {. }
$$

The skewness and kurtosis measures can be calculated from the central moments using the well-known relationships. To examine how the mean $\left(\mu_{1}^{\prime}\right)$, variance $\left(\sigma_{1}^{2}\right)$, skewness (Sk) and kurtosis $(\mathrm{Ku})$ change for various values of parameters $b, \lambda, a$ and $\beta$, numerical values are obtained. Table 1 contains the mean, variance, $\mathrm{Sk}$ and $\mathrm{Ku}$ of the WIL distribution for various values of parameters $b, \lambda, a$ and $\beta$. It can be noticed that both the mean and variance are non-decreasing for various values of $b, \lambda, a$ and $\beta$. Also, it can be noticed that both the Sk and the $\mathrm{Ku}$ are decreasing for various values of $b, \lambda, a$ and $\beta$.

Table 1: Mean, variance, skewness and kurtosis of WIL distribution for various values of $b, \lambda, a$ and $\beta$

\begin{tabular}{|c|c|cc|cc|cc|cc|}
\hline \multirow{3}{*}{$b$} & $\lambda$ & $a=0.5$ & $\beta=2$ & $a=0.5$ & $\beta=4.5$ & $a=0.5$ & $\beta=2$ & $a=0.5$ & $\beta=4.5$ \\
\cline { 2 - 10 } & & $\mu_{1}^{\prime}$ & $\sigma_{1}^{2}$ & $\mu_{1}^{\prime}$ & $\sigma_{1}^{2}$ & Sk & $\mathrm{Ku}$ & Sk & $\mathrm{Ku}$ \\
\hline \multirow{3}{*}{3} & 2 & 2.186 & 5.694 & 4.918 & 28.827 & 0.452 & 1.692 & 0.452 & 1.692 \\
\cline { 2 - 10 } & 2.5 & 2.842 & 9.524 & 6.395 & 48.215 & 0.428 & 1.650 & 0.428 & 1.650 \\
\cline { 2 - 10 } & 3 & 3.501 & 14.349 & 7.876 & 72.641 & 0.412 & 1.622 & 0.412 & 1.622 \\
\hline \multirow{3}{*}{3.5} & 2 & 2.202 & 5.552 & 4.954 & 28.105 & 0.354 & 1.508 & 0.354 & 1.508 \\
\cline { 2 - 10 } & 2.5 & 2.862 & 9.304 & 6.440 & 47.101 & 0.334 & 1.477 & 0.334 & 1.477 \\
\cline { 2 - 10 } & 3 & 3.525 & 14.035 & 7.931 & 71.052 & 0.321 & 1.457 & 0.321 & 1.457 \\
\hline \multirow{3}{*}{4} & 2 & 2.217 & 5.474 & 4.988 & 27.714 & 0.285 & 1.392 & 0.285 & 1.392 \\
\cline { 2 - 10 } & 2.5 & 2.881 & 9.187 & 0.269 & 46.507 & 0.269 & 1.368 & 0.269 & 1.368 \\
\cline { 2 - 10 } & 3 & 3.548 & 13.869 & 7.983 & 70.214 & 0.258 & 1.353 & 0.258 & 1.353 \\
\hline \multirow{3}{*}{5} & 2 & 2.243 & 5.411 & 5.046 & 27.395 & 0.197 & 1.257 & 0.197 & 1.257 \\
\cline { 2 - 9 } & 2.5 & 2.914 & 9.094 & 6.556 & 46.04 & 0.185 & 1.241 & 0.185 & 1.241 \\
\cline { 2 - 9 } & 3 & 3.587 & 13.743 & 8.072 & 69.574 & 0.178 & 1.231 & 0.178 & 1.231 \\
\hline
\end{tabular}


Recall the Taylor's series expansion of the function $e^{t x}$, that is $e^{t x}=\sum_{s=0}^{\infty} \frac{(t x)^{s}}{s !}$, so the moment generating function of the WIL distribution for $|t|<1$, is given by

$M_{x}(t)=\sum_{i, j, s=0}^{\infty} w_{i, j} \frac{(\beta t)^{s} \Gamma(1-s) \Gamma(s+\lambda(b+b i+j))}{\Gamma(\lambda(b+b i+j)) s !}, s=1,2, \ldots$,

The $k^{\text {th }}$ inverse moment, for the WIL distribution is derived by using pdf (9) as follows

$E\left(\frac{1}{X^{k}}\right)=\sum_{i, j=0}^{\infty} \beta^{-k} \frac{\Gamma(1+k) \Gamma(\lambda(b+b i+j)-k)}{\Gamma(\lambda(b+b i+j)+1)}, k=1,2, \ldots$,

The harmonic mean of the WIL distribution can be obtained by using the first inverse moment.

\subsection{Rényi Entropy}

The entropy of a random variable provides an excellent tool to quantify the amount of information (or uncertainty) contained in a random observation regarding its parent distribution (population). A large value of entropy implies the greater uncertainty in the data (Rényi, 1961). The concept of entropy is important in various situations in science, engineering and economics. The Rényi entropy of a random variable $X$ is defined by

$$
I_{R}(x)=(1-\rho)^{-1} \log \left(\int_{0}^{\infty}(f(x))^{\rho} d x\right),
$$

for $\rho>0$, and $\rho \neq 1$. The Rényi entropy of the WIL distribution is obtained by inserting the pdf (5) in (11) as follows

$I_{R}(x)=\frac{1}{(1-\rho)} \log \int_{0}^{\infty}(a b \lambda)^{\rho} \beta^{-\rho \lambda b} x^{\rho(\lambda b-1)}\left(1+\frac{x}{\beta}\right)^{-\rho(\lambda b+1)}\left(1-\left(1+\frac{\beta}{x}\right)^{-\lambda}\right)^{-\rho(b+1)} e^{\left[-a \rho\left[\frac{\left(1+\frac{\beta}{x}\right)^{-\lambda}}{1-\left(1+\frac{\beta}{x}\right)^{-\lambda}}\right]^{b}\right.} d x$.

After simplification, the Rényi entropy of WIL can be expressed as follows

$$
I_{R}(x)=\frac{1}{(1-\rho)} \log \left((a b \lambda)^{\rho} \beta^{1-\rho} \sum_{i, j=0}^{\infty} \frac{(-a \rho)^{j} \Gamma(\rho(b+1)+b j+i)}{\Gamma(\rho(b+1)+b j) i ! j !} \mathrm{B}((2 \rho-1)+1, \lambda(b j+b i+\rho b)-\rho+1)\right) .
$$

\subsection{Distribution of Order Statistics}

A closed form expression for the pdf of the $u^{\text {th }}$ order statistics of the WIL distribution is derived. Let $X_{(1)}<X_{(2)}, \ldots,<X_{(n)}$ be the order statistics for a random sample $X_{1}, X_{2}, \ldots, X_{\mathrm{n}}$ of size $n$ from WIL distribution. It is known that, the pdf of the $u^{\text {th }}$ order statistic is defined by

$f_{X_{(u)}}(x ; \psi)=\frac{1}{B(u, n-u+1)} \sum_{p=0}^{n-u}(-1)^{p}\left(\begin{array}{c}n-u \\ p\end{array}\right)[F(x ; \psi)]^{u+p-1} f(x ; \psi)$.

Inserting pdf (9) and cdf (4) in (12) and after simplification, we obtain the pdf of $u^{\text {th }}$ order statistic of WIL distribution as follows 


$$
\begin{aligned}
f_{X_{(u)}}(x ; \psi) & =\sum_{p=0}^{n-u} \sum_{k=0}^{u+p-1} \sum_{i, j, m, h=0}^{\infty} \zeta_{p, k, i, j, m, h} b \lambda x^{\lambda(b+b i+j)-1}\left(1+\frac{x}{\beta}\right)^{-(\lambda(b+b i+j)+1)}\left(1+\frac{\beta}{x}\right)^{-(\lambda b m+h \lambda)}, \\
\zeta_{p, k, i, j, m, h} & =\left(\begin{array}{c}
n-u \\
p
\end{array}\right)\left(\begin{array}{c}
u+p-1 \\
k
\end{array}\right) \frac{(-1)^{i+k+m+p} a^{i+1+m} k^{m} \Gamma(b+b i+1+j) \Gamma(b m+h)}{\Gamma(b+b i+1) i ! j ! m ! \Gamma(b m) h !} .
\end{aligned}
$$

The pdf of the smallest and largest order statistic of WIL distribution is obtained by setting $u=1$ and $u=n$ in (13), respectively, as follows

$$
\begin{aligned}
f_{X_{(1)}}(x ; \psi) & =\sum_{p=0}^{n-1} \sum_{k=0}^{p} \sum_{i, j, m, h=0}^{\infty} \varpi_{p, k, i, j, m, h} b \lambda x^{\lambda(b+b i+j)-1}\left(1+\frac{x}{\beta}\right)^{-(\lambda(b+b i+j)+1)}\left(1+\frac{\beta}{x}\right)^{-(\lambda b m+h \lambda)}, \\
\varpi_{p, k, i, j, m, h} & =\left(\begin{array}{c}
n-1 \\
p
\end{array}\right)\left(\begin{array}{l}
p \\
k
\end{array}\right) \frac{(-1)^{i+k+m+p} a^{i+1+m} k^{m} \Gamma(b+b i+1+j) \Gamma(b m+h)}{\Gamma(b+b i+1) i ! j ! m ! \Gamma(b m) h !},
\end{aligned}
$$

and,

$$
\begin{gathered}
f_{X_{(n)}}(x ; \psi)=\sum_{k=0}^{n+p-1} \sum_{i, j, m, h=0}^{\infty} \omega_{k, i, j, m, h} b \lambda x^{\lambda(b+b i+j)-1}\left(1+\frac{x}{\beta}\right)^{-(\lambda(b+b i+j)+1)}\left(1+\frac{\beta}{x}\right)^{-(\lambda b m+h \lambda)}, \\
\omega_{k, i, j, m, h}=\left(\begin{array}{c}
u+n-1 \\
k
\end{array}\right) \frac{(-1)^{i+k+m+p} a^{i+1+m} k^{m} \Gamma(b+b i+1+j) \Gamma(b m+h)}{\Gamma(b+b i+1) i ! j ! m ! \Gamma(b m) h !} .
\end{gathered}
$$

\subsection{Quantile Function}

The quantile function of $X$, where $X \sim \operatorname{WIL}(x ; \psi)$ is obtained by inverting $X_{Q}=F^{-1}(Q)$ as

$$
X_{Q}=\beta\left(\left[\left(-\frac{1}{a} \ln (1-Q)\right)^{\frac{-1}{b}}+1\right]^{\frac{1}{\lambda}}-1\right)^{-1}, \quad 0<Q<1 \text {. }
$$

Setting $Q=0.5$ in (14) gives the median of $X$. Simulating the WIL random variable is straightforward. If $Q$ is a uniform variate on the unit interval $(0,1)$, then the random variable $X$ follows (5).

\section{Estimation of the Model Parameters}

In this section, ML estimators of the population parameters for WIL distribution are derived based on Type II censored samples. ML, reliability function estimators and approximate confidence interval (CI) for the population parameters are obtained.

Let $n$ items, whose life time's follow the WIL distribution (5) are put on test. The test is terminated when the $r^{\text {th }}$ item fails for some fixed value of $r$. The lifetimes of these first $r$ failed items, say $X_{(1)}<X_{(2)}<\ldots<X_{(r)}$ are observed. The log likelihood function of the WIL distribution is obtained as follows 


$$
\begin{gathered}
L(\lambda, a, b, \beta, \underline{x})=\frac{n !}{(n-r) !} \prod_{i=1}^{r} a b \lambda \beta^{-\lambda b} x_{(i)}^{\lambda b-1}\left(1+\frac{x_{(i)}}{\beta}\right)^{-(\lambda b+1)}\left(1-\left(1+\frac{\beta}{x_{(i)}}\right)^{-\lambda}\right)^{-(b+1)} e^{-a\left[\left(1+\frac{\beta}{x_{(i)}}\right)^{\lambda}-1\right]^{-b}} \times \\
\left(e^{-a\left[\left(1+\frac{\beta}{x_{(i)}}\right)^{\lambda}-1\right]^{-b}}\right)^{n-r} .
\end{gathered}
$$

For simplicity, we write $x_{i}$ instead of $x_{(i)}$. The log likelihood function of the WIL distribution, denoted by $\ln l$, is obtained as follows

$$
\begin{aligned}
\ln l= & \ln (n ! /(n-r) !)+r \ln a+r \ln \lambda+r \ln b-r \lambda b \ln \beta+(\lambda b-1) \sum_{i=1}^{r} \ln \left(x_{i}\right)-(\lambda b+1) \sum_{i=1}^{r} \ln \left(1+\frac{x_{i}}{\beta}\right) \\
& -(b+1) \sum_{i=1}^{r} \ln \left(1-E_{i}\right)-a \sum_{i=1}^{r}\left[D_{i}-1\right]^{-b}-(n-r) a\left[D_{r}-1\right]^{-b},
\end{aligned}
$$

where, $D_{i}=\left(1+\frac{\beta}{x_{i}}\right)^{\lambda}, E_{i}=\left(1+\frac{\beta}{x_{i}}\right)^{-\lambda}$. The components of the score vector $(\partial \ln l / \partial \lambda$, $\partial \ln l / \partial a, \partial \ln l / \partial b, \partial \ln l / \partial \beta)$ are obtained as follows

$$
\begin{aligned}
\frac{\partial \ln l}{\partial \lambda} & =\frac{r}{\lambda}-b r \ln \beta+b \sum_{i=1}^{r} \ln x_{i}-b \sum_{i=1}^{r} \ln \left(1+\frac{x_{i}}{\beta}\right)-(b+1) \sum_{i=1}^{r}\left(D_{i}-1\right)^{-1} \ln \left(1+\frac{\beta}{x_{i}}\right) \\
& +b a \sum_{i=1}^{r}\left[D_{i}-1\right]^{-(b+1)} D_{i} \ln \left(1+\frac{\beta}{x_{i}}\right)+(n-r) a b\left[D_{r}-1\right]^{-(b+1)} D_{r} \ln \left(1+\frac{\beta}{x_{i}}\right), \\
\frac{\partial \ln l}{\partial b}= & \frac{r}{b}-\lambda r \ln \beta+\lambda \sum_{i=1}^{r} \ln x_{i}-\lambda \sum_{i=1}^{r} \ln \left(1+\frac{x_{i}}{\beta}\right)-\sum_{i=1}^{r} \ln \left(1-E_{i}\right)+a \sum_{i=1}^{r}\left[D_{i}-1\right]^{-b} \ln \left[D_{i}-1\right] \\
& +(n-r) a\left[D_{r}-1\right]^{-b} \ln \left[D_{r}-1\right],
\end{aligned}
$$

and,

$$
\begin{aligned}
\frac{\partial \ln l}{\partial \beta} & =\frac{-\lambda b r}{\beta}+(\lambda b+1) \sum_{i=1}^{r} \frac{x_{i} \beta}{\left(\beta+x_{i}\right)}+(b+1) \lambda \sum_{i=1}^{r} \frac{E_{i}^{1+\frac{1}{\lambda}}}{x_{i}\left(1-E_{i}\right)}+a b \lambda \sum_{i=1}^{r}\left[D_{i}-1\right]^{-(b+1)} \frac{D_{i}^{1-\frac{1}{\lambda}}}{x_{i}} \\
& +(n-r) a b \lambda\left[D_{r}-1\right]^{-(b+1)} \frac{D_{r}^{1-\frac{1}{\lambda}}}{x_{r}},
\end{aligned}
$$

The ML estimators of the unknown parameters of the WIL distribution can be obtained by solving the following non-linear equations: $\partial \ln l / \partial \lambda=0, \partial \ln l / \partial a=0, \partial \ln l / \partial b=0$ and $\partial \ln l / \partial \beta=0$. Unfortunately these equations cannot be solved analytically and can be solved numerically. 
For interval estimation of the parameters, the $4 \times 4$ observed information matrix $I(\psi)=\left\{I_{w x}\right\}$ for $(w, x)=(\lambda, a, b, \beta)$, are obtained. Under the regularity conditions, the known asymptotic properties of the ML method ensure that:

$\sqrt{n}(\hat{\psi}-\psi) \stackrel{d}{\longrightarrow} N_{4}\left(0, I^{-1}(\psi)\right)$ as $n \rightarrow \infty$ where $\stackrel{d}{\longrightarrow}$ means the convergence in distribution, with mean $\underline{0}=(0,0,0,0)^{T}$ and $4 \times 4$ covariance matrix $I^{-1}(\psi)$ then, the approximate $100(1-\tau) \%$ two sided CIs for $\lambda, a, b, \beta$, are respectively, given by:

$\hat{\lambda} \pm Z_{\tau / 2} \sqrt{\operatorname{var}(\hat{\lambda})}, \hat{a} \pm Z_{\tau / 2} \sqrt{\operatorname{var}(\hat{a})}, \hat{b} \pm \sqrt{\operatorname{var}(\hat{b})}, \hat{\beta} \pm Z_{\tau / 2} \sqrt{\operatorname{var}(\hat{\beta})}$.

Here, $Z_{\tau / 2}$ is the upper $\tau / 2^{\text {th }}$ percentile of the standard normal distribution and var (.)'s denote the diagonal elements of $I^{-1}(\psi)$ corresponding to the model parameters.

\section{Simulation Study}

In this section, numerical study is presented to illustrate the performance of the estimates for different parameter values and at two censoring levels. The performance of the estimates of unknown parameters and the reliability function are measured in terms of their mean square errors (MSEs), bias, standard errors (SEs), lower confidence bound (LCB), upper confidence bound (UCB), and the length of 95\%, CI. The numerical procedures are defined through the following algorithm.

Step (1): 1000 random samples of size $n=50,100$ and 150 are selected; these random samples are generated from the WIL distribution.

Step (2): The number of failure items; $r$, based on two levels of censoring schemes, is selected at $90 \%$ and $70 \%$ censoring levels.

Step (3): Different values of the unknown parameters $(\lambda, a, b, \beta)$ are selected as as Set $1 \equiv$ $(\lambda=0.5, a=2, b=0.5, \beta=0.7), \operatorname{Set} 2 \equiv(\lambda=0.7, a=1.8, b=0.8, \beta=0.5), \operatorname{Set} 3 \equiv$ $(\lambda=0.9, a=2.2, b=0.5, \beta=0.7)$ and Set4 $\equiv(\lambda=0.7, a=1.6, b=0.5, \beta=0.5)$.

Step (4): For all sample sizes and for parameters values; the MSE, bias, and SE are computed.

Step (5): The LCB, UCB and length of CIs with confidence level 0.95 for all samples sizes and parameters values are computed.

Step (6): ML estimates and 95\% CI for reliability function at the different mission time's $t_{0}$ where $\left(t_{0}=0.1,0.3\right.$ and 0.6$)$ for different sample sizes and level of censoring $r=0.9 n$ are calculated.

Numerical results are outlined in Tables 2 to 7 . From these tables, the following observations can be detected about the performance of the estimated parameters:

- For all set of parameters, SE of all estimated parameters decreases as $n$ increases. Also, the SEs for all set of parameters at censoring level $90 \%$ are smaller than the corresponding at censoring level 70\% (see Tables 2 and 3).

- The MSE and bias of $\lambda, a, b$ and $\beta$ decrease as $n$ increases for all values of parameters. Also, the MSE and bias for the numbers of the failure items at $r=0.7 n$ for all parameters values are the larger than the corresponding at $r=0.9 n$ (see Tables 2 and 3 ). 
- The MSEs and SEs of $\lambda$ and $\beta$ are smaller than the corresponding MSEs and SEs for the other estimates for $a$ and $b$ in most of situations (see Tables 2 and 3 ).

- For the same sample size, for fixed value of $\lambda=0.5, \beta=0.7$ and as the value of $a$ increases, the MSE and bias of $a$ increase (see Table 2).

- The length of CIs for the population parameters decreases as $n$ increases (see Tables 4 and 5).

- The MSE for the Set 4 has the smallest values corresponding to other sets.

- The ML estimates of reliability function decrease as the mission time's increase for all values of parameters.

- The length of CI gets shorter when $n$ increases (see Tables 4, 5, 6 and 7).

- The MSE of all ML estimates decreases as the censoring level increases for all sets of parameters (see for examples Figures 3 and 4).

- The ML estimates decrease as the censoring level increases. Also, the Set 1 has the smallest MSE compared to the other sets.

- The MSE of ML estimates of Set 3 at censoring level $90 \%$ decreases as $n$ increases (see Figure 5). Also, the MSE of ML estimates of Set 2 at censoring level $70 \%$ decreases as $n$ increases (see Figure 6 ).

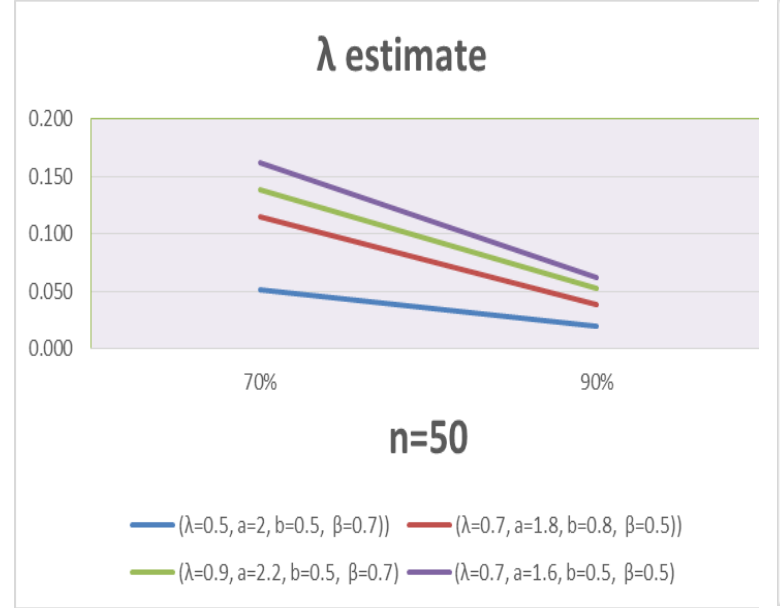

Figure 3: MSEs of $\lambda$ for all set of parameters

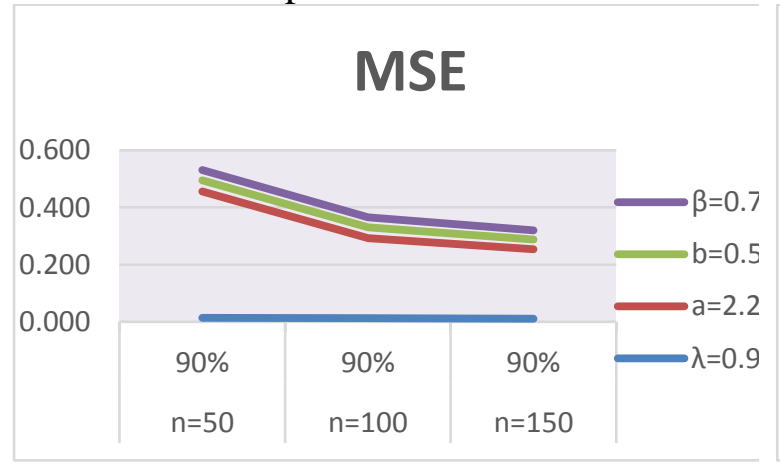

Figure 5: MSEs of Set 3 at $90 \%$ level of censoring

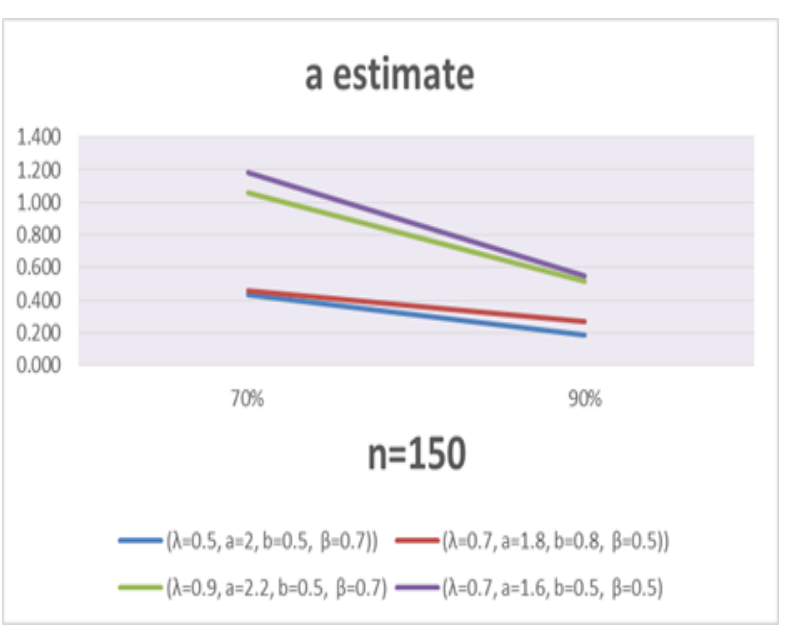

Figure 4: MSEs of $a$ for all set of parameters

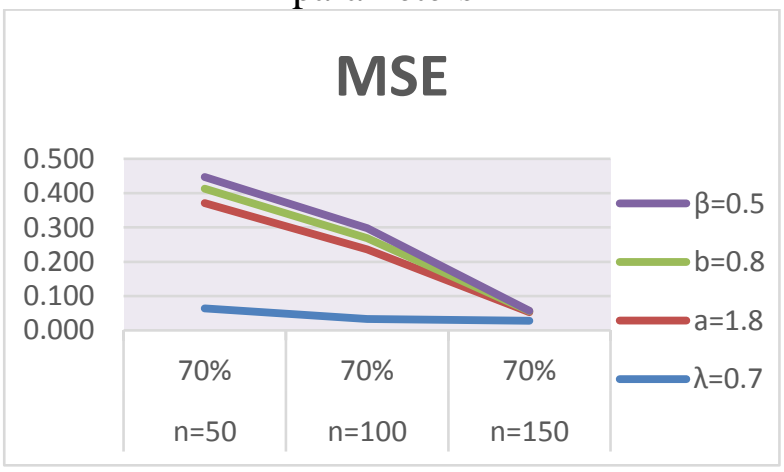

Figure6: MSEs of Set2 at 70\% level of censoring 
Table 2: The MSE, bias and SE of the estimates for Set 1 and Set 2

\begin{tabular}{|c|c|c|c|c|c|c|c|c|c|c|}
\hline \multirow[b]{2}{*}{$n$} & \multirow[b]{2}{*}{$r$} & \multirow[b]{2}{*}{ Properties } & \multicolumn{4}{|c|}{$(\lambda=0.5, a=2, b=0.5, \beta=0.7)$} & \multicolumn{4}{|c|}{$(\lambda=0.7, a=1.8, b=0.8, \beta=0.5)$} \\
\hline & & & $\hat{\lambda}$ & $\hat{a}$ & $\hat{b}$ & $\hat{\beta}$ & $\hat{\lambda}$ & $\hat{a}$ & $\hat{b}$ & $\hat{\beta}$ \\
\hline \multirow{6}{*}{50} & \multirow{3}{*}{35} & MSE & 0.051 & 0.632 & 0.268 & 0.265 & 0.064 & 0.307 & 0.042 & 0.034 \\
\hline & & Bias & 0.207 & -0.772 & -0.052 & -0.515 & 0.239 & -0.547 & 0.002 & -0.184 \\
\hline & & $\mathrm{SE}$ & 0.002 & 0.004 & 0.001 & 0.000 & 0.002 & 0.002 & 0.002 & 0.000 \\
\hline & \multirow{3}{*}{45} & MSE & 0.020 & 0.392 & 0.143 & 0.139 & 0.019 & 0.184 & 0.016 & 0.008 \\
\hline & & Bias & 0.108 & -0.584 & -0.022 & -0.373 & 0.119 & -0.417 & 0.047 & -0.086 \\
\hline & & SE & 0.002 & 0.005 & 0.001 & 0.000 & 0.001 & 0.002 & 0.002 & 0.005 \\
\hline \multirow{6}{*}{100} & \multirow{3}{*}{70} & MSE & 0.044 & 0.471 & 0.245 & 0.244 & 0.033 & 0.203 & 0.033 & 0.029 \\
\hline & & Bias & 0.190 & -0.654 & -0.078 & -0.493 & 0.168 & -0.441 & -0.023 & -0.167 \\
\hline & & SE & 0.001 & 0.002 & 0.001 & 0.000 & 0.007 & 0.001 & 0.001 & 0.000 \\
\hline & \multirow{3}{*}{90} & MSE & 0.014 & 0.287 & 0.119 & 0.117 & 0.015 & 0.135 & 0.017 & 0.013 \\
\hline & & Bias & 0.088 & -0.495 & -0.035 & -0.341 & 0.096 & -0.338 & -0.004 & -0.106 \\
\hline & & SE & 0.001 & 0.002 & 0.001 & 0.000 & 0.001 & 0.001 & 0.001 & 0.000 \\
\hline \multirow{6}{*}{150} & \multirow{3}{*}{105} & MSE & 0.039 & 0.437 & 0.232 & 0.232 & 0.028 & 0.025 & 0.003 & 0.001 \\
\hline & & Bias & 0.183 & -0.630 & -0.083 & -0.480 & 0.155 & 0.023 & -0.029 & 0.017 \\
\hline & & SE & 0.001 & 0.001 & 0.000 & 0.000 & 0.000 & 0.001 & 0.000 & 0.000 \\
\hline & \multirow{3}{*}{135} & MSE & 0.004 & 0.183 & 0.026 & 0.026 & 0.014 & 0.086 & 0.005 & 0.000 \\
\hline & & Bias & 0.048 & -0.387 & -0.009 & -0.155 & 0.055 & -0.102 & 0.012 & -0.005 \\
\hline & & SE & 0.000 & 0.001 & 0.000 & 0.000 & 0.007 & 0.002 & 0.001 & 0.000 \\
\hline
\end{tabular}

Table 3: The MSE, bias and SE of the estimates for Set 3 and Set 4

\begin{tabular}{|c|c|c|c|c|c|c|c|c|c|c|}
\hline \multirow[b]{2}{*}{$n$} & \multirow[b]{2}{*}{$r$} & \multirow{2}{*}{ Properties } & \multicolumn{4}{|c|}{$(\lambda=0.9, a=2.2, b=0.5, \beta=0.7)$} & \multicolumn{4}{|c|}{$(\lambda=0.7, a=1.6, b=0.5, \beta=0.5)$} \\
\hline & & & $\hat{\lambda}$ & $\hat{a}$ & $\hat{b}$ & $\hat{\beta}$ & $\hat{\lambda}$ & $\hat{a}$ & $\hat{b}$ & $\hat{\beta}$ \\
\hline \multirow{6}{*}{50} & \multirow{3}{*}{35} & MSE & 0.023 & 0.983 & 0.150 & 0.145 & 0.024 & 0.238 & 0.035 & 0.030 \\
\hline & & Bias & 0.113 & -0.984 & 0.870 & -0.381 & 0.118 & -0.465 & 0.047 & -0.174 \\
\hline & & SE & 0.002 & 0.003 & 0.002 & 0.000 & 0.002 & 0.003 & 0.001 & 0.000 \\
\hline & \multirow{3}{*}{45} & MSE & 0.014 & 0.442 & 0.039 & 0.036 & 0.009 & 0.071 & 0.005 & 0.007 \\
\hline & & Bias & 0.082 & -0.654 & 0.068 & -0.189 & 0.047 & -0.222 & 0.057 & 0.026 \\
\hline & & $\mathrm{SE}$ & 0.002 & 0.002 & 0.001 & 0.002 & 0.002 & 0.003 & 0.001 & 0.000 \\
\hline \multirow{6}{*}{100} & \multirow{3}{*}{70} & MSE & 0.020 & 0.704 & 0.138 & 0.136 & 0.019 & 0.147 & 0.031 & 0.029 \\
\hline & & Bias & 0.091 & -0.817 & 0.039 & -0.368 & 0.107 & -0.362 & 0.009 & -0.169 \\
\hline & & SE & 0.001 & 2 & 01 & 0.000 & 0.008 & 0.001 & 0.001 & 0.000 \\
\hline & \multirow{3}{*}{90} & MSE & 0.013 & 0.279 & 0.038 & 0.036 & 0.009 & 0.054 & 0.004 & 0.003 \\
\hline & & Bias & 0.056 & -0.495 & 0.034 & -0.190 & 0.025 & -0.164 & 0.026 & -0.018 \\
\hline & & SE & 0.001 & 0.002 & 0.001 & 0.000 & 0.001 & 0.002 & 0.001 & 0.000 \\
\hline \multirow{6}{*}{150} & \multirow{3}{*}{105} & MSE & 0.012 & 0.597 & 0.127 & 0.125 & 0.015 & 0.128 & 0.027 & 0.025 \\
\hline & & Bias & 0.061 & -0.761 & 0.028 & -0.354 & 0.087 & -0.324 & -0.004 & -0.159 \\
\hline & & SE & 0.001 & 0.001 & 0.000 & 0.000 & 0.001 & 0.001 & 0.000 & 0.000 \\
\hline & \multirow{3}{*}{135} & MSE & 0.011 & 0.243 & 0.034 & 0.032 & 0.008 & 0.034 & 0.003 & 0.001 \\
\hline & & Bias & 0.028 & -0.453 & 0.025 & -0.177 & 0.008 & -0.093 & 0.024 & 0.028 \\
\hline & & $\mathrm{SE}$ & 0.001 & 0.001 & 0.000 & 0.000 & 0.001 & 0.001 & 0.000 & 0.000 \\
\hline
\end{tabular}


Table 4: LCB, UCB and Length of the estimates for Set 1 and Set 2

\begin{tabular}{|c|c|c|c|c|c|c|c|c|}
\hline \multirow{2}{*}{$n$} & \multirow{2}{*}{$r$} & \multirow{2}{*}{ Parameters } & \multicolumn{3}{|c|}{$(\lambda=0.5, a=2, b=0.5, \beta=0.7)$} & \multicolumn{3}{|c|}{$(\lambda=0.7, a=1.8, b=0.8, \beta=0.5)$} \\
\hline & & & LCB & UCB & Length & LCB & UCB & Length \\
\hline \multirow{8}{*}{50} & \multirow{4}{*}{35} & $\lambda$ & 0.703 & 0.710 & 0.007 & 0.936 & 0.942 & 0.007 \\
\hline & & $\mathrm{a}$ & 1.221 & 1.236 & 0.015 & 1.250 & 1.258 & 0.008 \\
\hline & & $\mathrm{b}$ & 0.445 & 0.450 & 0.004 & 0.800 & 0.808 & 0.007 \\
\hline & & $\beta$ & 0.185 & 0.448 & 0.263 & 0.315 & 0.805 & 0.490 \\
\hline & \multirow{4}{*}{45} & $\lambda$ & 0.605 & 0.612 & 0.007 & 0.816 & 0.822 & 0.005 \\
\hline & & $\mathrm{a}$ & 1.407 & 1.425 & 0.018 & 1.379 & 1.387 & 0.008 \\
\hline & & $\mathrm{b}$ & 0.476 & 0.480 & 0.004 & 0.843 & 0.850 & 0.007 \\
\hline & & $\beta$ & 0.327 & 0.478 & 0.151 & 0.413 & 0.848 & 0.434 \\
\hline \multirow{8}{*}{100} & \multirow{4}{*}{70} & $\lambda$ & 0.691 & 0.694 & 0.003 & 0.867 & 0.869 & 0.003 \\
\hline & & $\mathrm{a}$ & 1.338 & 1.346 & 0.008 & 1.459 & 1.465 & 0.006 \\
\hline & & b & 0.422 & 0.424 & 0.002 & 0.775 & 0.778 & 0.003 \\
\hline & & $\beta$ & 0.211 & 0.423 & 0.212 & 0.333 & 0.777 & 0.445 \\
\hline & \multirow{4}{*}{90} & $\lambda$ & 0.591 & 0.595 & 0.003 & 0.794 & 0.797 & 0.003 \\
\hline & & $\mathrm{a}$ & 1.495 & 1.504 & 0.008 & 1.357 & 1.361 & 0.004 \\
\hline & & $\mathrm{b}$ & 0.464 & 0.466 & 0.002 & 0.795 & 0.798 & 0.003 \\
\hline & & $\beta$ & 0.350 & 0.465 & 0.114 & 0.798 & 0.797 & 0.404 \\
\hline \multirow{8}{*}{150} & \multirow{4}{*}{105} & $\lambda$ & 0.682 & 0.684 & 0.002 & 0.854 & 0.856 & 0.002 \\
\hline & & $\mathrm{a}$ & 1.367 & 1.372 & 0.005 & 1.821 & 1.825 & 0.004 \\
\hline & & b & 0.417 & 0.418 & 0.001 & 0.770 & 0.772 & 0.001 \\
\hline & & $\beta$ & 0.219 & 0.417 & 0.198 & 0.517 & 0.771 & 0.300 \\
\hline & \multirow{4}{*}{135} & $\lambda$ & 0.577 & 0.579 & 0.002 & 0.754 & 0.756 & 0.002 \\
\hline & & $\mathrm{a}$ & 1.501 & 1.505 & 0.005 & 1.698 & 1.702 & 0.004 \\
\hline & & $\mathrm{b}$ & 0.461 & 0.463 & 0.001 & 0.811 & 0.813 & 0.002 \\
\hline & & $\beta$ & 0.357 & 0.462 & 0.106 & 0.494 & 0.813 & 0.300 \\
\hline
\end{tabular}


Table 5: LCB, UCB and Length of the estimates for Set 3 and Set 4

\begin{tabular}{|c|c|c|c|c|c|c|c|c|}
\hline \multirow{2}{*}{$n$} & \multirow{2}{*}{$r$} & \multirow{2}{*}{ Parameters } & \multicolumn{3}{|c|}{$(\lambda=0.9, a=2.2, b=0.5, \beta=0.7)$} & \multicolumn{3}{|c|}{$(\lambda=0.7, a=1.6, b=0.5, \beta=0.5)$} \\
\hline & & & LCB & $\mathrm{UCB}$ & Length & LCB & $\mathrm{UCB}$ & Length \\
\hline \multirow{8}{*}{50} & \multirow{4}{*}{35} & $\lambda$ & 1.009 & 1.017 & 0.008 & 0.814 & 0.822 & 0.008 \\
\hline & & $\mathrm{a}$ & 1.211 & 1.221 & 0.010 & 1.129 & 1.141 & 0.012 \\
\hline & & $\mathrm{b}$ & 0.584 & 0.589 & 0.005 & 0.544 & 0.549 & 0.005 \\
\hline & & $\beta$ & 0.319 & 0.587 & 0.268 & 0.326 & 0.547 & 0.221 \\
\hline & \multirow{4}{*}{45} & $\lambda$ & 0.978 & 0.985 & 0.007 & 0.743 & 0.750 & 0.006 \\
\hline & & $\mathrm{a}$ & 1.541 & 1.550 & 0.009 & 1.372 & 1.383 & 0.011 \\
\hline & & $\mathrm{b}$ & 0.566 & 0.570 & 0.005 & 0.555 & 0.560 & 0.005 \\
\hline & & $\beta$ & 0.511 & 0.568 & 0.058 & 0.526 & 0.557 & 0.032 \\
\hline \multirow{8}{*}{100} & \multirow{4}{*}{70} & $\lambda$ & 0.989 & 0.993 & 0.004 & 0.806 & 0.809 & 0.003 \\
\hline & & $\mathrm{a}$ & 1.380 & 1.387 & 0.008 & 1.236 & 1.241 & 0.005 \\
\hline & & b & 0.538 & 0.540 & 0.002 & 0.508 & 0.510 & 0.002 \\
\hline & & $\beta$ & 0.332 & 0.540 & 0.208 & 0.331 & 0.510 & 0.179 \\
\hline & \multirow{4}{*}{90} & $\lambda$ & 0.954 & 0.958 & 0.004 & 0.724 & 0.727 & 0.003 \\
\hline & & $\mathrm{a}$ & 1.702 & 1.709 & 0.007 & 1.433 & 1.438 & 0.005 \\
\hline & & b & 0.533 & 0.535 & 0.002 & 0.525 & 0.527 & 0.002 \\
\hline & & $\beta$ & 0.510 & 0.535 & 0.025 & 0.524 & 0.526 & 0.002 \\
\hline \multirow{8}{*}{150} & \multirow{4}{*}{105} & $\lambda$ & 0.960 & 0.962 & 0.002 & 0.785 & 0.788 & 0.002 \\
\hline & & $\mathrm{a}$ & 1.436 & 1.441 & 0.005 & 1.274 & 1.278 & 0.004 \\
\hline & & $\mathrm{b}$ & 0.527 & 0.528 & 0.001 & 0.499 & 0.500 & 0.001 \\
\hline & & $\beta$ & 0.346 & 0.528 & 0.182 & 0.341 & 0.500 & 0.159 \\
\hline & \multirow{4}{*}{135} & $\lambda$ & 0.927 & 0.929 & 0.003 & 0.707 & 0.709 & 0.002 \\
\hline & & $\mathrm{a}$ & 1.745 & 1.750 & 0.005 & 1.504 & 1.509 & 0.004 \\
\hline & & $\mathrm{b}$ & 0.524 & 0.525 & 0.001 & 0.524 & 0.525 & 0.001 \\
\hline & & $\beta$ & 0.522 & 0.525 & 0.003 & 0.526 & 0.528 & 0.002 \\
\hline
\end{tabular}

Table 6: ML estimates and 95\% CI of reliability function for Set 1 and Set 2 at censoring level $r=0.9 n$

\begin{tabular}{|c|c|c|c|c|c|c|c|c|c|c|}
\hline \multicolumn{7}{|c|}{$(\lambda=0.5, a=2, b=0.5, \beta=0.7)$} & \multicolumn{4}{|c|}{$(\lambda=0.7, a=1.8, b=0.8, \beta=0.5)$} \\
\hline \multirow{2}{*}{$n$} & \multirow{2}{*}{$r$} & \multirow{2}{*}{$t_{0}$} & \multirow{2}{*}{ average } & \multicolumn{3}{|c|}{$95 \% \mathrm{CI}$} & \multirow{2}{*}{ average } & \multicolumn{3}{|c|}{$95 \% \mathrm{CI}$} \\
\hline & & & & LCB & UCB & Length & & LCB & UCB & Length \\
\hline \multirow{3}{*}{50} & \multirow{3}{*}{45} & 0.1 & 0.972 & 0.948 & 0.996 & 0.048 & 1.000 & 1.000 & 1.000 & 0.000 \\
\hline & & 0.3 & 0.803 & 0.765 & 0.840 & 0.075 & 0.998 & 0.997 & 1.000 & 0.003 \\
\hline & & 0.6 & 0.510 & 0.473 & 0.548 & 0.075 & 0.972 & 0.959 & 0.985 & 0.026 \\
\hline \multirow{3}{*}{100} & \multirow{3}{*}{90} & 0.1 & 0.995 & 0.994 & 0.996 & 0.002 & 0.996 & 0.986 & 1.007 & 0.022 \\
\hline & & 0.3 & 0.729 & 0.711 & 0.747 & 0.036 & 0.996 & 0.983 & 1.008 & 0.024 \\
\hline & & 0.6 & 0.518 & 0.498 & 0.537 & 0.038 & 0.981 & 0.968 & 0.995 & 0.026 \\
\hline \multirow{3}{*}{150} & \multirow{3}{*}{135} & 0.1 & 0.995 & 0.994 & 0.996 & 0.002 & 0.998 & 0.992 & 1.004 & 0.012 \\
\hline & & 0.3 & 0.721 & 0.707 & 0.735 & 0.028 & 0.998 & 0.991 & 1.005 & 0.014 \\
\hline & & 0.6 & 0.527 & 0.507 & 0.548 & 0.041 & 0.998 & 0.985 & 0.991 & 0.007 \\
\hline
\end{tabular}


Table 7: ML estimates and 95\% CI of reliability function for Set 3 and Set 4 at censoring level $r=0.9 n$

\begin{tabular}{|c|c|c|c|c|c|c|c|c|c|c|}
\hline \multicolumn{7}{|c|}{$(\lambda=0.9, a=2.2, b=0.5, \beta=0.7)$} & \multicolumn{4}{|c|}{$(\lambda=0.7, a=1.6, b=0.5, \beta=0.5)$} \\
\hline \multirow{2}{*}{$n$} & \multirow{2}{*}{$r$} & \multirow{2}{*}{$t_{0}$} & \multirow{2}{*}{ average } & \multicolumn{3}{|c|}{$95 \% \mathrm{CI}$} & \multirow{2}{*}{ average } & \multicolumn{3}{|c|}{$95 \% \mathrm{CI}$} \\
\hline & & & & LCB & UCB & Length & & LCB & UCB & Length \\
\hline \multirow{3}{*}{50} & \multirow{3}{*}{45} & 0.1 & 0.996 & 0.986 & 1.005 & 0.018 & 0.999 & 0.998 & 1.000 & 0.002 \\
\hline & & 0.3 & 0.956 & 0.936 & 0.976 & 0.040 & 0.902 & 0.879 & 0.925 & 0.047 \\
\hline & & 0.6 & 0.804 & 0.765 & 0.843 & 0.078 & 0.600 & 0.555 & 0.644 & 0.089 \\
\hline \multirow{3}{*}{100} & \multirow{3}{*}{90} & 0.1 & 0.994 & 0.990 & 0.998 & 0.009 & 0.996 & 0.991 & 1.001 & 0.010 \\
\hline & & 0.3 & 0.920 & 0.906 & 0.933 & 0.028 & 0.921 & 0.908 & 0.934 & 0.026 \\
\hline & & 0.6 & 0.729 & 0.706 & 0.752 & 0.046 & 0.686 & 0.663 & 0.709 & 0.046 \\
\hline \multirow{3}{*}{150} & \multirow{3}{*}{135} & 0.1 & 0.996 & 0.995 & 0.998 & 0.003 & 0.998 & 0.996 & 1.000 & 0.004 \\
\hline & & 0.3 & 0.928 & 0.921 & 0.936 & 0.016 & 0.926 & 0.918 & 0.933 & 0.016 \\
\hline & & 0.6 & 0.732 & 0.716 & 0.747 & 0.031 & 0.687 & 0.671 & 0.702 & 0.031 \\
\hline
\end{tabular}

\section{Applications}

In this section, two real data sets are provided to illustrate the importance of the WIL distribution. The first data set refers to Hinkley (1977), which consists of thirty successive values of March precipitation (in inches) in Minneapolis/St Paul. The data are listed as follows: $0.77,1.74,0.81,1.20,1.95,1.20,0.47,1.43,3.37,2.20,3.00,3.09$, $1.51,2.10,0.52,1.62,1.31,0.32,0.59,0.81,2.81,1.87,1.18,1.35,4.75,2.48,0.96,1.89$, $0.90,2.05$

The second data refer to Jorgensen (1982) will be considered, it consists of 40 observations of the active repair times (in hours) for airborne communication transceiver. The data are: $0.50,0.60,0.60,0.70,0.70,0.70,0.80,0.80,1.00,1.00,1.00,1.00,1.10$, $1.30,1.50,1.50,1.50,1.50,2.00,2.00,2.20,2.50,2.70,3.00,3.00,3.30,4.00,4.00,4.50$, $4.70,5.00,5.405 .40,7.00,7.50,8.80,9.00,10.20,22.00,24.50$.

To check the validity of the fitted model, Kolmogorov-Smirnov goodness of fit test and its p-value are obtained. The estimated pdf and cdf for both data are provided in Figures 7 and 8. It is clear from these figures that the WIL distribution fitting these both data sets. 

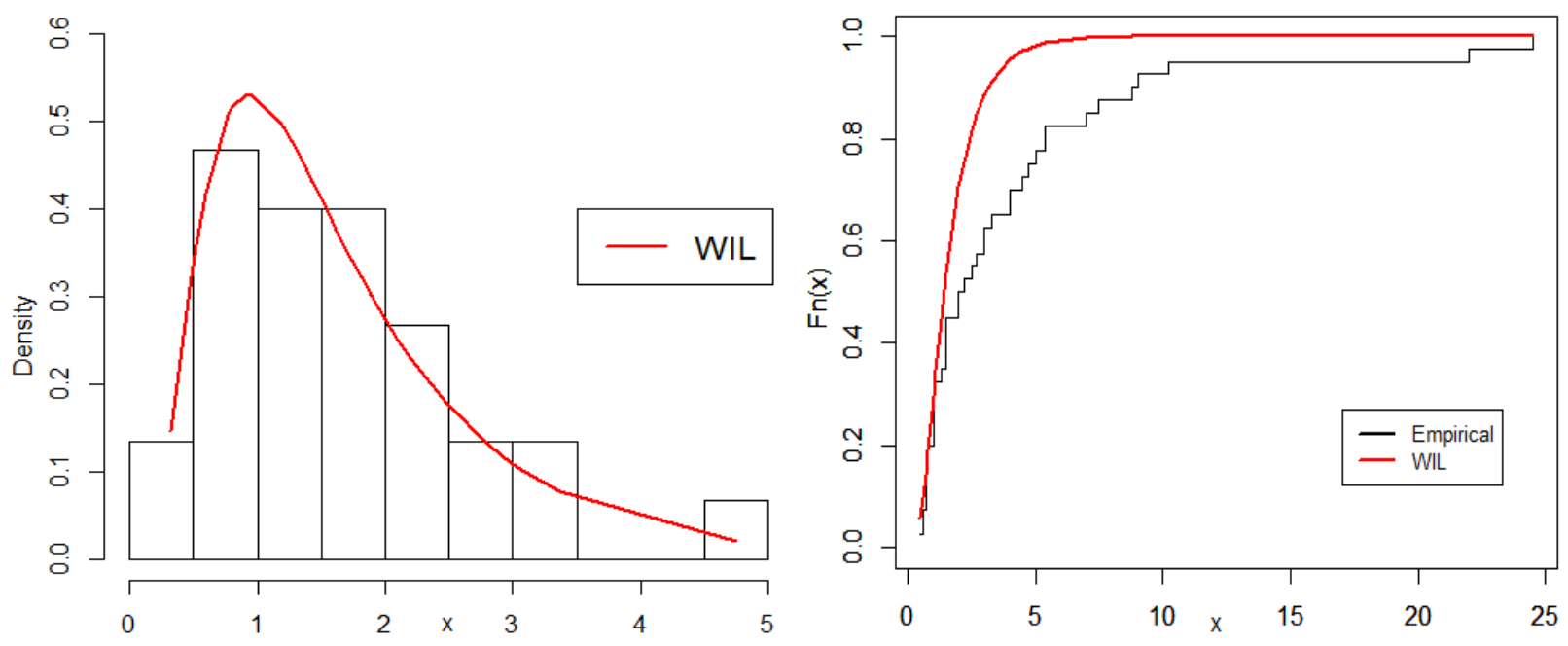

Figure 7: Estimated pdf and cdf of the WIL model for the first data
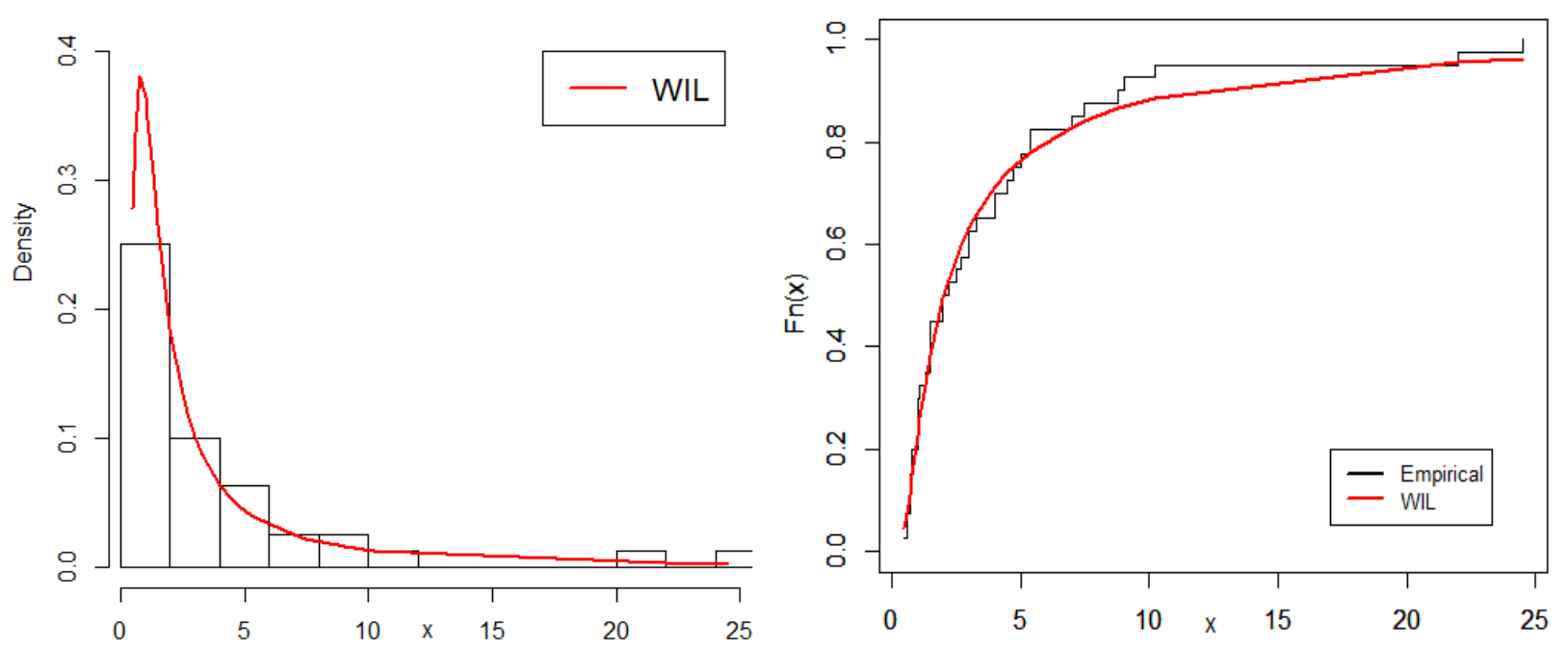

Figure 8: Estimated pdf and cdf of the WIL model for the second data

Furthermore, the ML estimates of the population parameters, reliability function and their SEs for both real data sets based on TIIC are listed in Table 8.

Table 8: ML estimate of the parameters, reliability function and their SEs Based on TIIC samples

\begin{tabular}{|c|c|c|c|c|c|c|c|c|c|c|c|}
\hline \multicolumn{7}{|c|}{ First Real Data } & \multicolumn{5}{|c|}{ Second Real Data } \\
\hline$n$ & $r$ & $t_{0}$ & estimator & estimate & SEs & $n$ & $r$ & $t_{0}$ & estimator & estimate & SEs \\
\hline \multirow{5}{*}{30} & \multirow{5}{*}{21} & \multirow{5}{*}{0.1} & $\hat{\lambda}$ & 7.59 & 0.272 & \multirow{5}{*}{40} & \multirow{5}{*}{28} & \multirow{5}{*}{0.1} & $\hat{\lambda}$ & 30.71 & 0.913 \\
\hline & & & $\hat{a}$ & 3.533 & 0.103 & & & & $\hat{a}$ & 1.86 & 0.022 \\
\hline & & & $\hat{b}$ & 0.873 & 0.019 & & & & $\hat{b}$ & 0.401 & 0.004 \\
\hline & & & $\hat{\beta}$ & 0.430 & 0.011 & & & & $\hat{\beta}$ & 0.176 & 0.004 \\
\hline & & & $\hat{R}\left(t_{0}\right)$ & 1 & & & & & $\hat{R}\left(t_{0}\right)$ & 1 & \\
\hline
\end{tabular}




\section{Concluding Remarks}

In this paper, a new distribution with four parameters, called the Weibull inverse Lomax distribution is introduced. Some of the statistical properties of the new distribution such as quantile function, moments, order statistics and Rényi entropy are obtained. Maximum likelihood estimators of population parameters are obtained based on Type II censored samples. The approximate confidence intervals for the model parameters and reliability function are derived. Simulation study is presented to illustrate the performance of estimates for two censoring schemes. Two real data sets are presented to illustrate theortical results.

\section{Acknowledgements}

The authors would like to thank the Editor and the anonymous referees, for their valuable and very constructive comments, which have greatly improved the contents of the paper.

\section{References}

1. Abouelmagd, T.H.M., Al-mualim, S., Elgarhy, M., Afify, A. Z., and Ahmad, M. (2017). Properties of the four-parameter Weibull distribution and its applications. Pakistan Journal of Statistics, 33(6), 449-466.

2. Alzaatreh, A., Lee, C. and Famoye, F. (2013). A new method for generating families of continuous distributions. Metron, 71, 63-79.

3. Bourguignon, M., Silva, R. B. and Cordeiro, G. M. (2014). The Weibull-G family of probability distributions. Journal of Data Science, 12, 53-68.

4. Eugene, N., Lee, C. and Famoye, F. (2002). The beta -normal distribution and its applications. Communications in Statistics-Theory and Methods, 31(4), 497-512.

5. Fisher, B. and Kilicman, A. (2012). Some results on the gamma function for negative integers. Applied Mathematics and Information Sciences, 6, 173-176.

6. Hassan, A. S., Elbatal, I. and Hemeda, S. E. (2016). Weibull quasi Lindley distribution and its statistical properties with applications to lifetime data. International Journal of Applied Mathematics and Statistics, 55(3), 63-80.

7. Hinkley, D. (1977).On Quick Choice of Power Transformations. Journal of the Royal Statistical Society, Series(c), Applied Statistics, 26(1), 67-69.

8. Ibrahim, N. A, Khaleel, M. A. Merovci, F., Kilicman, A. and Shitan, M. (2017). Weibull Burr $\mathrm{X}$ distribution properties and application. Pakistan Journal of Statistics, 33(5), 315-336.

9. Jones, M. C. (2004). Families of distributions arising from distributions of order statistics. Test, 13, 1-43.

10. Jorgensen, B. (1982). Statistical properties of the generalized inverse Gaussian distribution, Springer-Verlag, New York.

11. Kleiber, C. (2004). Lorenz ordering of order statistics from log-logistic and related distributions. Journal of Statistical Planning and Inference, 120, 13-19.

12. Kleiber, C., and Kotz, S. (2003). Statistical size distributions in economics and actuarial sciences. John Wiley and Sons, Inc., Hoboken, New Jersey. 
13. McKenzie, D., Miller, C., and Falk, D. A. (2011). The Landscape Ecology of Fire. Springer Dordrecht Heidelberg, New York.

14. Merovci, F. and Elbatal, I. (2015). Weibull Rayleigh distribution: theory and applications. Applied Mathematics and Information Sciences, 5(9), 1-11

15. Rahman, J., and Aslam, M. (2014). Interval prediction of future order statistics in two-component mixture inverse Lomax model: a Bayesian approach. American Journal of Mathematical and Management Science, 33, 216-227.

16. Rahman, J., and Aslam, M. (2017). On estimation of two-component mixture inverse Lomax model via Bayesian approach. International Journal of System Assurance Engineering and Management, 8, 99-109.

17. Rahman, J., Aslam, M., and Ali, S. (2013). Estimation and prediction of inverse Lomax model via Bayesian Approach. Caspian Journal of Applied Sciences Research, 2(3), 43-56.

18. Rényi A. (1961). On measures of entropy and information. In: Proceedings of the $4^{\text {th }}$ Fourth Berkeley Symposium on Mathematical Statistics and Probability, 547561. University of California Press, Berkeley

19. Reyad, H. M., and Othman, S. A. (2018). E- Bayesian estimation of twocomponent mixture of inverse Lomax distribution based on type- I censoring scheme, Journal of Advances in Mathematics and Computer Science (26) 2, 1-22.

20. Singh, S. K., Singh, U. and Yadav, A. S. (2016). Reliability estimation for inverse Lomax distribution under type $\Pi$ censored data using Markov chain Monte Carlo method. International Journal of Mathematics and Statistics, 17(1), 128-146.

21. Tahir, M. H., Cordeiro, G. M., Mansoor, M. and Zubair, M., (2015). The WeibullLomax distribution: properties and applications. Hacettepe Journal of Mathematics and Statistics, 44 (2), $461-480$

22. Yadav, A. S., Singh, S. K., and Singh, U. (2016). On hybrid censored inverse Lomax distribution: application to the survival data. Statistica, 76(2), 185-203. 